\title{
NUMERICAL RELATIVITY SIMULATIONS OF MAGNETIZED BLACK HOLE-NEUTRON STAR MERGERS
}

\author{
ZACHARIAH B. ETIENNE*, YUK TUNG LIU, VASILEIOS PASCHALIDIS, and \\ STUART L. SHAPIRO \\ Department of Physics, University of Illinois, \\ Urbana, IL 61801-3080, USA \\ *E-mail: zetienne@illinois.edu \\ www.illinois.edu
}

\begin{abstract}
We present new numerical techniques ${ }^{1}$ we developed for launching the first parameter study of magnetized black hole-neutron star (BHNS) mergers, varying the magnetic fields seeded in the initial neutron star. We found that magnetic fields have a negligible impact on the gravitational waveforms and bulk dynamics of the system during merger, regardless of magnetic field strength or $\mathrm{BH}$ spin. In a recent simulation, we seeded the remnant disk from an unmagnetized BHNS merger simulation with large-scale, purely poloidal magnetic fields, which are otherwise absent in the full simulation. The outcome appears to be a viable sGRB central engine.
\end{abstract}

Keywords: Compact Binary Mergers, Compact Objects, Neutron Star, Black Hole, General Relativity, Numerical Relativity, Magnetohydrodynamics

\section{Evolving the Equations of General Relativistic Magnetohydrodynamics on Adaptively-Refined Grids}

We have recently extended our adaptive mesh refinement (AMR) numerical relativity code to solve the ideal magnetohydrodynamics (MHD) equations, enabling us to simulate MHD effects in dynamical spacetimes with AMR ${ }^{112}$ The subtlety in evolving these equations is enforcing the divergence-free constraint $\boldsymbol{\nabla} \cdot \boldsymbol{B}=0$. If we were to evolve the induction equation in the most obvious and straightforward way, numerical errors will lead to violation of this divergence-free constraint, resulting in the production of spurious magnetic monopoles. There are several known solutions to this problem in unigrid simulations, but few when AMR is used. The one we chose was to evolve the vector potential, $\mathcal{A}^{\mu}$. In this case, the magnetic induction and divergence-free equations in curved spacetime become:

$$
\begin{aligned}
B^{i} & =\epsilon^{i j k} \partial_{j} A_{k}, \\
\partial_{t} A_{i} & =\epsilon_{i j k} v^{j} B^{k}-\partial_{i}\left(\alpha \Phi-\beta^{j} A_{j}\right) \\
\partial_{j} \tilde{B}^{j} & =\partial_{i}\left(\tilde{\epsilon}^{i j k} \partial_{j} A_{k}\right) \equiv 0,
\end{aligned}
$$

where $B^{i}=\tilde{B}^{i} / \sqrt{\gamma}$ is the magnetic field measured by a normal observer, $A_{\mu}=\mathcal{A}_{\mu}-$ $\Phi n_{\mu}$ is the projection of the four-vector potential $\mathcal{A}_{\mu}$ onto a 3 -dimensional spacelike hypersurface, $\Phi$ the scalar potential, $n^{\mu}$ is the normal vector to the hypersurface, $\tilde{\epsilon}^{i j k}=\epsilon^{i j k} / \sqrt{\gamma}, \epsilon^{i j k}=n_{\mu} \epsilon^{\mu i j k}$ is the 3-dimensional Levi-Civita tensor associated with the 3-metric $\gamma_{i j}$, and $\gamma$ the 3-metric determinant.

By construction, we guarantee in Eq. 3 that the divergence of the magnetic field is zero, since the divergence of a curl is zero. This property is guaranteed, no 
matter what interpolation scheme we choose when interpolating between different adaptively refined grids.

When evolving the vector potential, an electromagnetic (EM) gauge choice must be made. In choosing an EM gauge, there is a subtlety. We have written our numerical prescription so that the resulting magnetic fields are completely invariant to the EM gauge choice inside uniform-resolution grids.

However, when we adaptively add subgrids at higher resolution using AMR, interpolation at mesh refinement boundaries turns EM gauge modes into physical modes, thereby affecting the magnetic fields. Thus, if we are not careful in our gauge choice, the gauge-dependent magnetic fields induced on these refinement boundaries may poison our simulation.

Our first attempt at a gauge condition was $\partial_{i}\left(\alpha \Phi-\beta^{j} A_{j}\right)=0$, as it greatly simplifies the right-hand side of Eq. 2. However, we later found that this gauge choice introduces a zero-speed gauge mode ${ }^{1}$ With this zero-speed mode, if the path of magnetized matter crosses an AMR refinement boundary, interpolation on this boundary leads to the creation of weak, spurious magnetic fields in black holeneutron star (BHNS) simulations that grow stronger with time until the simulation crashes.

So we switched from our original choice to the Lorenz gauge $\nabla_{\mu} \mathcal{A}^{\mu}=0$, in which the EM gauge modes propagate away, thereby drastically reducing the appearance of spurious magnetic fields at refinement boundaries. The simulations presented in the next section were the first to use this gauge for full GRMHD with AMR.

\section{Magnetized Black Hole-Neutron Star Binary Mergers}

As a neutron star (NS) is tidally disrupted by a black hole (BH) companion at the end of a BHNS binary inspiral, its magnetic fields will be stretched, wound, and amplified. If sufficiently strong, these magnetic fields may impact the gravitational waveforms, merger evolution and mass of the remnant disk. Formation of highly-collimated magnetic field lines in the disk+spinning $\mathrm{BH}$ remnant may launch relativistic jets, providing the central engine for a short-hard GRB (sGRB). We explore this scenario through fully general relativistic, magnetohydrodynamic (GRMHD) BHNS simulations from inspiral through merger and disk formation.2 In particular, we attempt to answer the following two questions:

(1) How do NS magnetic fields affect BHNS binary waveforms and the resulting $\mathrm{BH}+$ disk system?

(2) Do we produce an sGRB progenitor?

To answer these questions, we perform simulations in which the $\mathrm{BH}$ is initially spinning with spin parameter 0.75 , aligned with the orbital angular momentum. Though surface NS magnetic field strengths have been inferred by observation, very little is known about NS interior magnetic fields. So for this preliminary investigation we seed only the NS interior with initially poloidal magnetic fields. The initial 
data are shown in the left panel of Fig. 1, Keeping this field configuration fixed, we vary the initial magnetic field strength, choosing magnetic fields with average magnetic to gas pressure $P_{\mathrm{B}} / P_{\text {gas }}$ of $0,5 \times 10^{-5}$, and $5 \times 10^{-3}$. Note that the case with the strongest magnetic fields has field strengths of order $10^{17} \mathrm{G}$ at the core of the NS (assuming the NS has a rest mass of $1.4 M_{\odot}$ ). We choose to seed the NS with magnetic fields sufficiently weak to avoid disturbing the NS equilibrium during inspiral, but sufficiently strong to influence the final outcome.

To address the first question, we find that magnetic fields have no significant impact on the gravitational waveforms or residual disk masses, regardless of initial strength. Magnetic fields retain their poloidal structure during the final orbit before merger. But in terms of magnetic field structure, there is a large difference between pre- and post-disruption: magnetic fields that were almost purely poloidal initially become almost purely toroidal due to the rapid winding of the matter around the $\mathrm{BH}$ as it forms a disk. One of the ingredients in sGRB models is the collimation of magnetic fields perpendicular to the disk. The right frame of Fig. 1 demonstrates the lack of magnetic field collimation in the $P_{\mathrm{B}} / P_{\text {gas }}=5 \times 10^{-3}$ case.
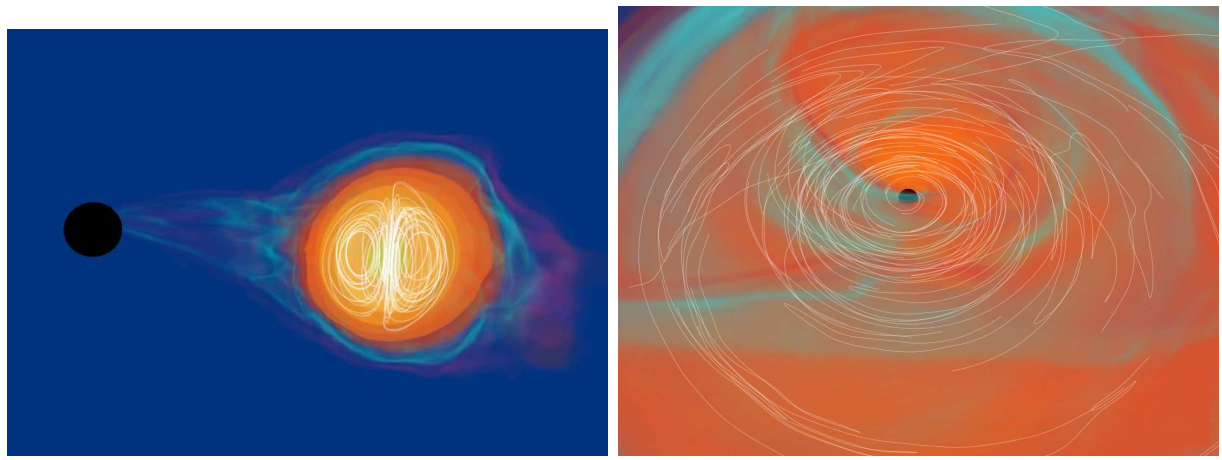

Fig. 1. 3D density and magnetic field snapshots. Left: initial data, NS on the right (from highest to lowest rest-mass density, the colors are: yellow, orange, red, and cyan), BH apparent horizon $(\mathrm{AH})$ on the left. Right: final disk density profile with magnetic field lines, about $33 \mathrm{~ms}\left(1.4 M_{\odot} / M_{0}\right)$ after disk formation $(t=2072 M)$, where $M_{0}$ is the initial rest mass of the NS and $M$ the ADM mass of the system.

We stopped our simulation about 30ms after tidal disruption. Notice that we had a thick disk orbiting a spinning $\mathrm{BH}$, but there was no strong evidence of magnetic field collimation. But what about relativistic outflows, another key ingredient for sGRB central engines? After 30 ms of disk evolution, we find no outflows: lowdensity matter is still rapidly accreting onto the $\mathrm{BH}$ poles and no sign of fluid velocity reversal is observed.

In our latest work,$[3]$ we demonstrated that when the remnant disk from an unmagnetized BHNS simulation is seeded with large-scale poloidal fields, we observe spectacular collimated magnetic fields and relativistic outflows, as shown in the panels of Fig. 2, However, such large-scale poloidal fields may be difficult to generate 


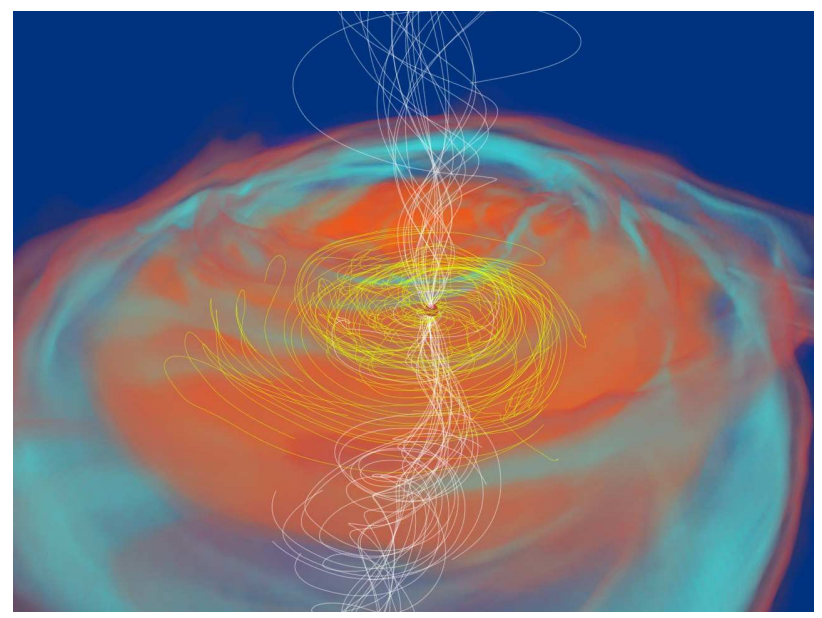

Fig. 2. 3D snapshot, corresponding to the case in which we seed a remnant disk from an unmagnetized BHNS simulation with purely poloidal magnetic fields. This is a snapshot taken when we terminate the simulation, viewing from above the disk plane. Magnetic field streamlines emerging just above and below the $\mathrm{BH}$ poles are shown in white, and those in the disk are shown in yellow.

in a fully self-consistent BHNS simulation, as the magnetic fields must follow the NS fluid as it wraps around the spinning BH during tidal disruption and disk formation, generating strong toroidal fields. GRMHD simulations performed by other groups indicate that $\mathrm{BH}$ accretion disks lacking large-scale poloidal fields may not

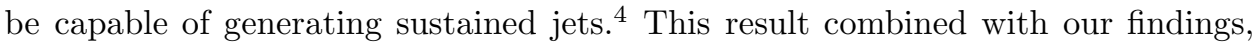
make BHNS mergers less likely sGRB central engines.

In spite of this, we found in this same work ${ }^{3}$ that inserting tilted magnetic fields into the NS breaks the initial equatorial symmetry of the problem and encourages poloidal fluid motion, resulting in 10x stronger poloidal magnetic fields in the remnant disk. Even with these stronger poloidal magnetic fields, no magnetic collimation or relativistic outflows were observed. We anticipate that large-scale poloidal fields might be produced in BHNS simulations with highly-spinning BHs that are misaligned with the orbital angular momentum. Such a system might explain quasiperiodic signals in observed sGRBs. ${ }^{5}$

\section{References}

1. Z. B. Etienne, V. Paschalidis, Y. T. Liu and S. L. Shapiro, Phys. Rev. D 85, p. 024013(January 2012).

2. Z. B. Etienne, Y. T. Liu, V. Paschalidis and S. L. Shapiro, Phys. Rev. D 85, p. 064029(March 2012).

3. Z. B. Etienne, V. Paschalidis and S. L. Shapiro, Phys. Rev. D 86, p. 084026(October 2012).

4. K. Beckwith, J. F. Hawley and J. H. Krolik, Astrophys. J. 678, 1180(May 2008).

5. N. Stone, A. Loeb and E. Berger, ArXiv e-prints (September 2012). 